\title{
A Dynamic Brain Atlas
}

\author{
D.L.G. Hill ${ }^{1}$, J.V. Hajnal' ${ }^{2}$, D. Rueckert ${ }^{3}$, S.M. Smith ${ }^{4}$, T. Hartkens ${ }^{1}$, and K. McLeish ${ }^{1}$ \\ ${ }^{1}$ Computational Imaging Science, King's College London \\ Derek.Hill@kcl.ac.uk \\ ${ }^{2}$ Imaging Sciences Department, Clinical Sciences Centre, Imperial College London \\ ${ }^{3}$ Computer Science Dept. Imperial College London \\ ${ }^{4}$ FMRIB, University of Oxford
}

\begin{abstract}
We describe a dynamic atlas that can be customized to an individual study subject in near-real-time. The atlas comprises 180 brain volumes each of which has been automatically segmented into grey matter, white matter and $\mathrm{CSF}$, and also non-rigidly registered to the Montreal BrainWeb reference dataset providing automatic delineation of brain structures of interest. To create a dynamic atlas, the user loads a study dataset (eg: a patient) and queries the atlas database to identify similar subjects. All selected database subjects are then aligned with the study subject using affine registration, and average tissue probability maps and structure delineations produced. The system can run on distributed data and distributed CPUs illustrating the potential of computational grids in medical image analysis.
\end{abstract}

\section{Introduction}

Neuro-imaging research is a successful example of web-enabled science. The web enables exchange of standardized datasets (atlases) $[1,2,3,4,5]$ and research software tools ${ }^{1}$ and facilitates collaboration between individual research sites. But you cannot analyze data on the web. The "web-science" paradigm involves downloading software and / or data, and running algorithms for data analysis locally. This limits collaboration, and is one of the main reasons why neuroimaging research papers typically involve small cohorts of research subjects collected by one site.

This pattern of research is fairly typical of web-enabled science. The web has facilitated certain types of collaboration, but large scale group working is difficult to achieve. Computational grids [6] have the potential to extend the functionality of the internet in ways that will make collaborative science involving large amounts of data and sophisticated algorithms much more straightforward. Scientific collaboration built on computational grids can go beyond web-science by transparently integrating data repositories, sophisticated algorithms and distributed computing.

Similar issues are also important in healthcare. Digital image archive systems are becoming more widespread in hospitals, and image data is being incorporated into multimedia patient records. This will make widespread computational analysis of clinical (as opposed to research) images possible for the first time. The capabilities

\footnotetext{
${ }^{1}$ See, for example: www.cma.mgh.harvard.edu/tools 
provided by computational grids could make it possible to use the huge quantity of distributed on-line patient images for decision support in diagnosis.

In this paper we describe a proof of concept demonstrator that illustrates this potential through a network application that constructs brain atlases on the fly from selected subsets of a cohort of subjects 180 subjects.

\section{Method}

Our approach involved the following stages: assembling the data, and loading it onto an internet accessible database'; implementation of a network interface to launch data processing that creates new data rather than just retrieving stored data.; pre-processing the images to label tissue types and structures of interest; interactive use of the system to synthesize brain atlases specific to any individual on the fly.

\section{Assembling the Data}

The data comprised 180 whole brain magnetic resonance (MR) images of normal controls and patients with a variety of non-space-occupying brain lesions. All images were $\mathrm{rf}$ spoiled T1 weighted gradient echo volume acquisitions acquired on a 1T MR scanner (Philips Medical Systems, Cleveland Ohio), interpolated to approximately $1 \mathrm{~mm}$ cubic voxels. All data was anonymised prior to transfer to the database.

The database, constructed using the open source MySQL (www.mysql.com), recorded subject age at scan date, gender, study group, and the URL of the image file. The database was automatically populated with the study data by parsing the filenames and image headers. Although we used a single server, different parts of the dataset could have been stored on servers at separate sites.

\section{Network Interface}

A TCL/TK interface incorporated into an interactive image viewer provides a userfriendly environment to query the database, transfer images using the secure grid protocol globus-url-copy (www.globus.org) and securely launch processing on a distributed computing cluster using globus and condor-g (www.cs.wisc.edu/condor).

\section{Data Preparation}

Database images were segmented into tissue types, and anatomical structures of interest delineated. The very substantial computation required for these automated analysis stages was carried out on computing hardware distributed across three sites (University of Oxford, King's College London and Imperial College London). The database was automatically populated with the analysis results.

\section{Tissue Type Segmentation}

Each brain volume was segmented into grey matter, white matter, and CSF using a statistical classifier implemented as part of the publicly available FSL package (www.fmrib.ox.ac.uk/fsl), running on a $16 \mathrm{CPU}$ alpha server with 16Gbytes of RAM. 


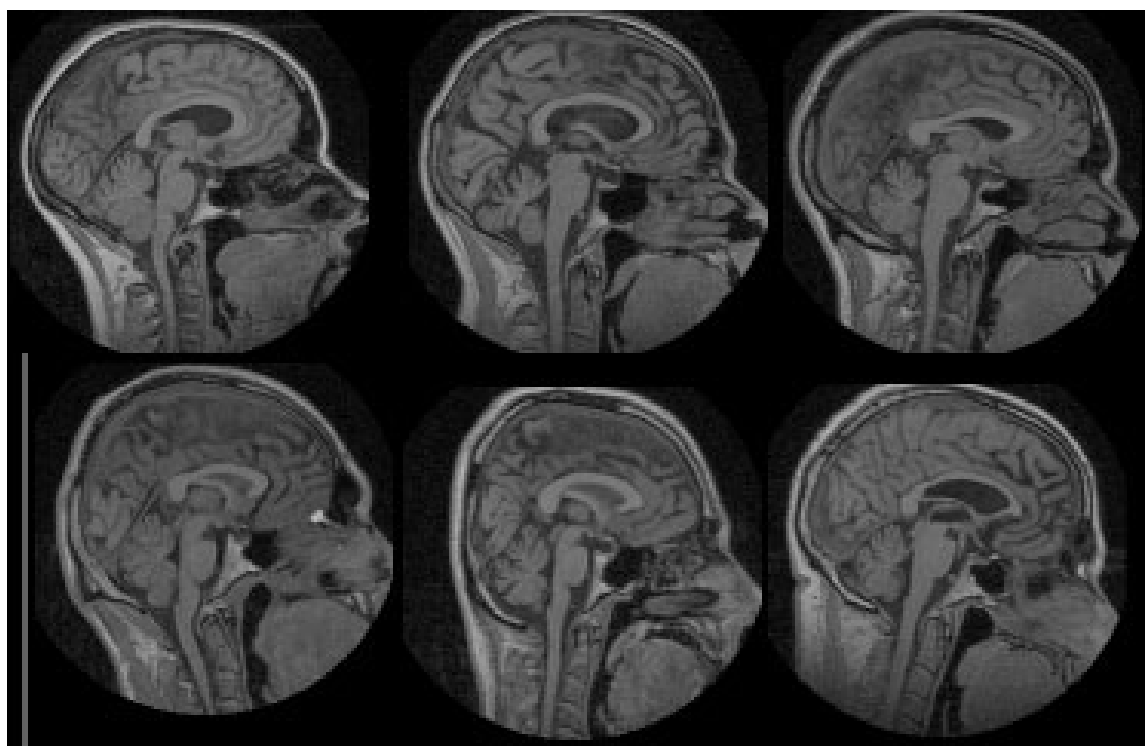

Fig. 1. Mid-sagittal sections through six of the 180 database subjects

\section{Anatomical Structure Delineation}

Anatomical structures were automatically delineated from the brain scans by registering the Montreal Brainweb reference image [9] to each scan in turn using a nonrigid registration algorithm based on manipulating a uniform array of B-spline control points (separation $2.4 \mathrm{~mm}$ ) while optimizing normalized mutual information [7]. The Brainweb reference image is implicitly segmented into grey matter, white matter and CSF (plus some other tissue types), and we had additionally delineated some anatomical structures including the caudate nucleus and lateral ventricles. After registration, the deformation field calculated was used to warp the segmented tissue labels and delineated structure boundaries to each of the 180 images, automatically segmenting and labeling each dataset to the accuracy of the registration algorithm. The non-rigid registration took 2 hours per dataset, and on a condor cluster of sixteen $1.4 \mathrm{GHz}$ Athlon PCs running linux, could process all 180 datasets in 24 hours.

\section{Interactive Use to Calculate Atlases}

The dynamic aspect of the system is the ability to generate an atlas specific to a particular subject in near real-time, rather than making use of a pre-computed atlas that may not be appropriate. The user loads a dataset of interest onto their local computer (we refer to this patient or research subject as the study subject). The user then queries the network database to identify database subjects that are close to the study subject in age, gender and/or other attributes stored in the database). The user then specifies the number of computers from the remote condor cluster they wish to make use of. Finally, the user launches the analysis. The study subject and instructions are then securely transferred to the computer cluster using globus protocols. Each of the 


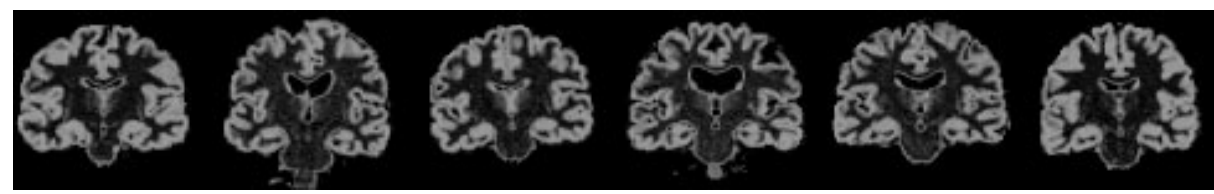

Fig. 2. Coronal sections through 6 example grey matter tissue probability maps selected from the 180 database subjects. Note intersubject variability

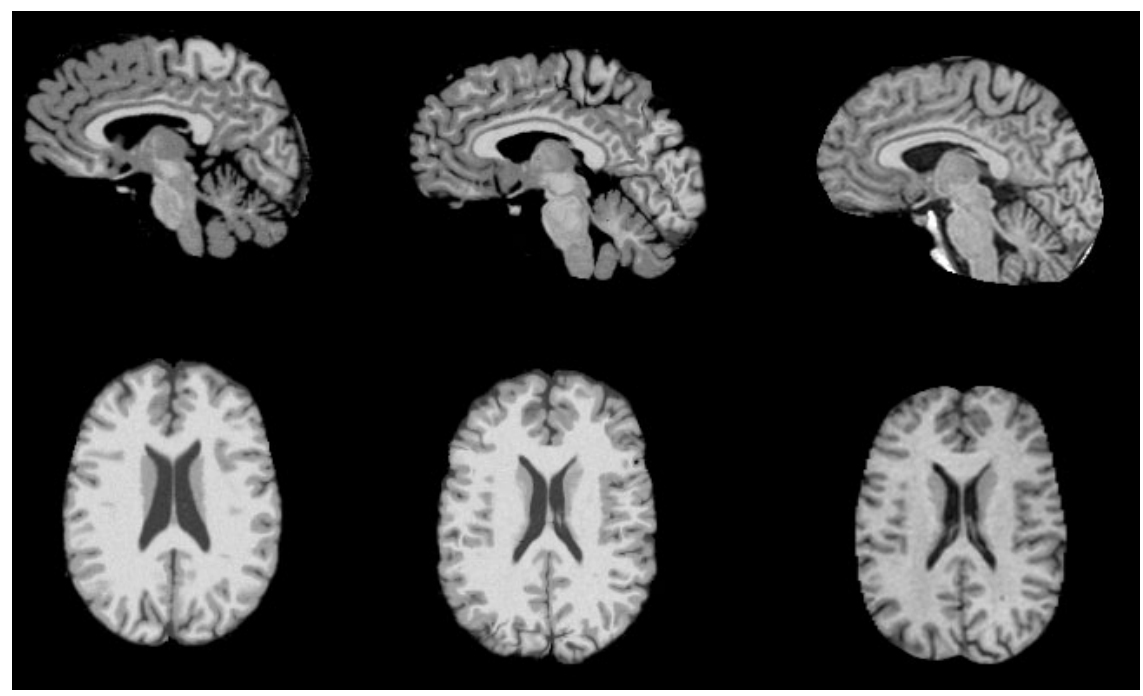

Fig. 3. Sagittal (top) and axial (bottom) slices through the Brainweb reference dataset (left), an example subject (right), and the reference dataset after warping to the subject (center). The reference dataset was warped to all 180 database subjects as part of pre-processing, effectively delineating all structures segmented in the reference dataset

selected database subjects is then registered to the study subject using an affine registration algorithm running on the condor cluster, and the resulting transformation is used to transform all the segmented tissue maps and delineated structures into the coordinate frame of the study subject and generate an atlas that comprises an average image of the tissue types and structure boundaries that can be used to assist in interpretation or subsequent analysis of the study subject. The results are securely transferred back to the local computer. On our cluster of 16 linux PCs, an atlas of up to 16 subjects could be generated in about 3 minutes.

\section{Results}

\section{Pre-processing}

Figure 1 shows some example sagittal sections through some of the 180 subjects in the database. Figure 2 shows example tissue probably maps for grey matter, for six 


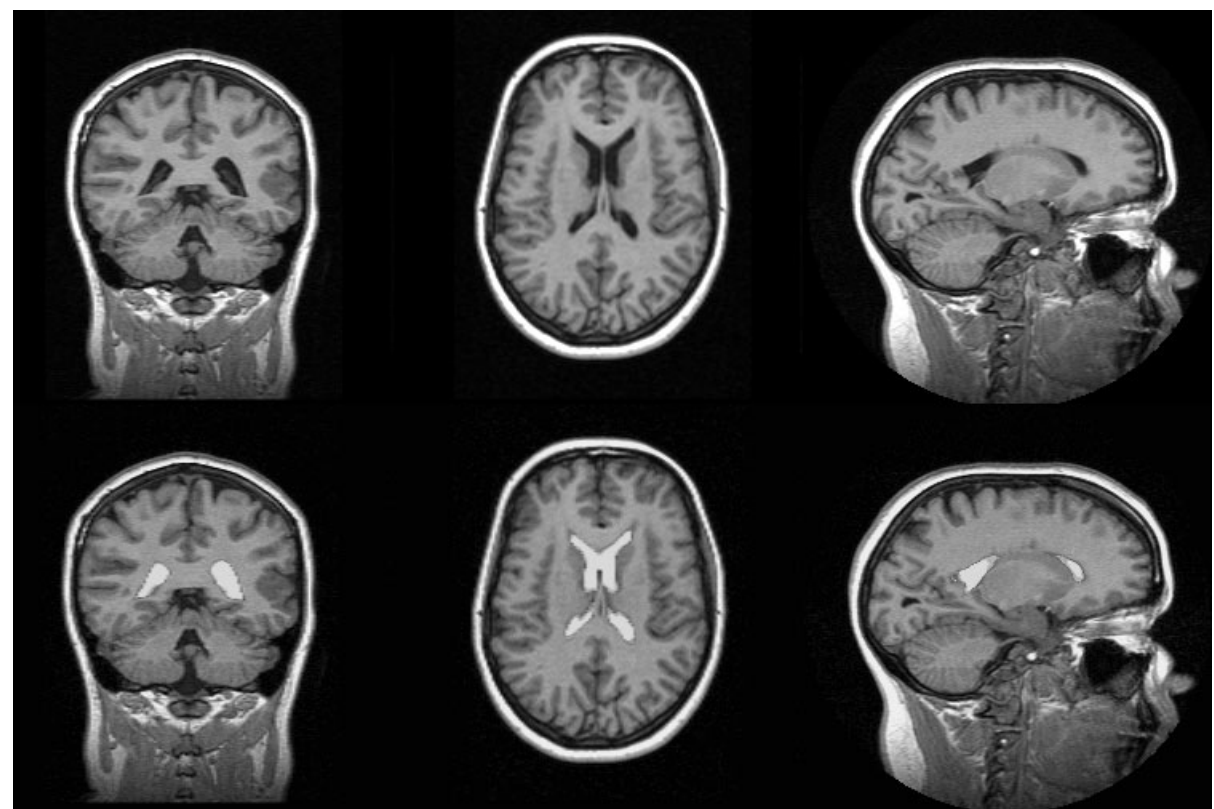

Fig. 4. Example coronal, axial and sagittal slices through a subject before and after delineation of the lateral ventricles using warping of the reference dataset

different subjects, reformatted in the coronal plane. Figure 3 shows an axial slice through the Brainweb reference dataset before, and after non-rigid registration to one of the subjects in the database. Figure 4 shows the subject image with the delineated structure warped from the reference dataset. These example results are shown as twodimensional slices, but the analysis was done in 3D on all 180 database subjects.

\section{Example Dynamically Generated Atlases}

Figures 5 shows CSF maps from example atlases generated for study subjects of three different ages: For a 25 year old subject, the atlas was generated from images in the database from 30 subjects between 16 and 35 years of age, each registered to the study subject, then with the pre-computed segmentations and delineations transformed into the study subject's coordinates and averaged. For a 45 year old subject, the process was repeated using database subjects between 35 and 65 years of age, and for a 75 year old subject, the atlas was generated from database subject over 70 years old. Although we show example images, the user can generate a new atlas by loading in a study subject of choice, querying the network database as they choose, and launching the data-analysis on the remote computer facility.

Figure 6 shows a study subject with the 50 percentile boundary of the lateral ventricles from a similar-aged group of database subjects overlaid. Similar overlays could be produced for any delineated structure in the reference image.

\section{Dicussion}

We have implemented a network application using computational grid protocols that allows the user to dynamically generate brain atlases customized to a selected study 


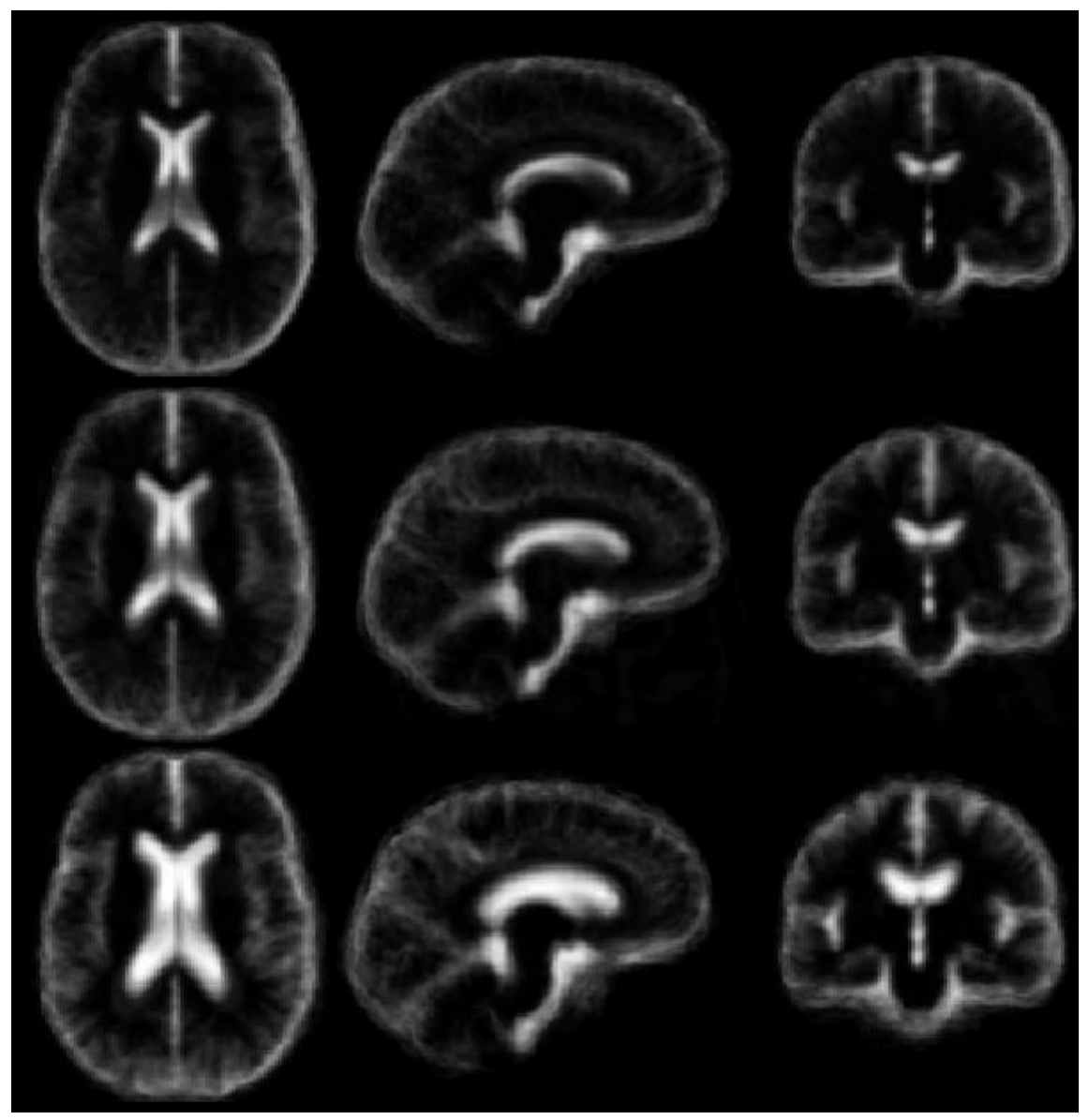

Fig. 5. CSF atlas produced for subjects aged 25 years (top), 45 years (middle) and 75 years (bottom). Note increasing size of CSF spaces with age. The dynamic atlas enables an atlas for any subject (selected by age or gender) to be calculated within a few minutes

subject. It provides a network interface that can be used to launch processing that creates new data, rather than just retrieving stored data. One of the characteristics of our network application and of grid applications in general is that they are scaleable, and it should be straightforward to extend them to much larger distributed collections of images and massive distributed computing facilities within multicentre collaborations. As the grid protocols evolve, it should make it increasingly easy to build and use large scale network applications of this type.

Dynamically configurable atlases have considerable value in neuroimaging research and in healthcare. In neuroimaging research, "static" web brain atlases are widely used to assist in data analysis and in cohort studies. A dynamic atlas could easily be configured to the research question of interest, which may provide added sensitivity to the analysis. In healthcare, MR imaging is being used more and more often in the management of patients with subtle or diffuse brain disease. Diagnosis of 


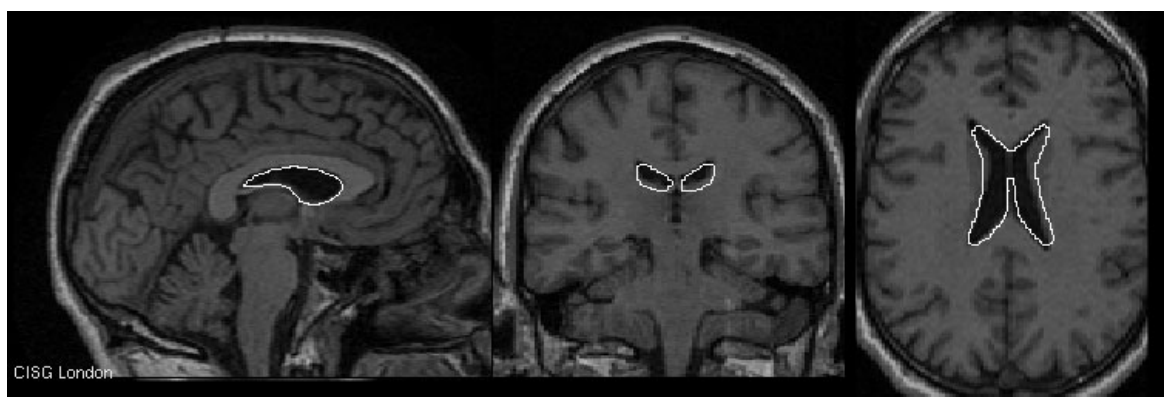

Fig. 6. Study subject with $50 \%$ boundary of lateral ventricles from age-mathed dynamic atlas overlaid as white boundary. This sort of functionality could provide decision support by assisting a radiologist in quantifying the degree of abnormality in a patient

such cases by conventional visual inspection of the images may be hard because of difficulties in precisely identifying what is abnormal about a subject's images. This process could be made more straightforward by providing an atlas made up of individuals of the same age, gender and possibly even co-morbidity as the study subject. Such an atlas could assist a radiologist by providing decision support that could in future be implemented on large healthcare image archives.

A natural extension of the dynamic atlas would be to add a knowledge discovery component. Rather than registering the images to a study subject, the network application could register the images to one-another in order to discover relationships between the data. The value of such a facility would increase with increasing numbers of subjects, and larger amounts of non-image information about the subjects, potentially including genetic information.

Future neuro-imaging research is going to require analysis of much larger cohorts of data in order to add to our understanding of brain development, aging and pathology. Such large scale projects will require better data analysis infrastructure than is provided by the existing world wide web and computing facilities at individual laboratories. We believe that this dynamic brain atlas project indicates the sort of capability that will be needed in such research programmes in the future.

\section{Acknowledgements}

This work was funded by the UK Department of Trade and Industry / Engineering and Physical Sciences Research Council e-science core programme as a technology demonstrator. We are grateful for the use of the MRI data, which was collected under the supervision of Professor Graeme Bydder, Dr Basant Puri and Dr Angela Oatridge. We thank Philips Medical Systems for supporting the MRI aspects of this work.

\section{References}

1. Sandor S. Leahy R. Surface-based labeling of cortical anatomy using a deformable atlas. IEEE Transactions on Medical Imaging. 16(1):41-54, 1997

2. Woods RP. Dapretto M. Sicotte NL. Toga AW. Mazziotta JC. Creation and use of a Talairach-compatible atlas for accurate, automated, nonlinear intersubject registration, and analysis of functional imaging data. Human Brain Mapping. 8(2-3):73-9, 1999 
3. van Leemput K. Maes F. Vandermeulen D. Suetens P. Automated model-based bias field correction of MR images of the brain. IEEE Transactions on Medical Imaging. 18(10):88596, 1999

4. Thompson PM. Mega MS. Woods RP. Zoumalan CI. Lindshield CJ. Blanton RE. Moussai J. Holmes CJ. Cummings JL. Toga AW. Cortical change in Alzheimer's disease detected with a disease-specific population-based brain atlas. Cerebral Cortex. 11(1):1-16, 2001

5. Chung MK. Worsley KJ. Paus T. Cherif C. Collins DL. Giedd JN. Rapoport JL. Evans AC. A unified statistical approach to deformation-based morphometry. Neuroimage. 14(3):595606, 2001

6. Foster, C. Kesselman, S. Tuecke The Anatomy of the Grid: Enabling Scalable Virtual Organizations.. International J. Supercomputer Applications, 15(3), 2001.

7. Rueckert D, Sonoda LI, Hayes C, Hill DLG, Leach MO, Hawkes DJ. Non-rigid Registration using Free-Form Deformations: Application to Breast MR Images IEEE Trans. Medical Imaging 18(8): 712-721 1999

8. D. L. Collins, A. P. Zijdenbos, V. Kollokian, J. Sled, N. J. Kabani, C. J. Holmes, and A. C. Evans. Design and construction of a realistic digital brain phantom. IEEE Transactions on Medical Imaging. 17,(3),463-468, 1998. 